\title{
Correlation and asymmetry between solar flare hard X-ray footpoints: a statistical study
}

\author{
M. Jin and M. D. Ding \\ Department of Astronomy, Nanjing University, Nanjing 210093, PR China \\ e-mail: dmd@nju.edu.cn \\ Received 31 January 2007 / Accepted 23 April 2007 \\ ABSTRACT

\begin{abstract}
Aims. We study the correlation and asymmetry between solar flare hard X-ray footpoint time profiles that can be used to constrain the energy release and particle acceleration processes in solar flares.

Methods. We perform a statistical analysis of correlations and asymmetries of hard X-ray footpoints for 29 solar flares that were observed by the Reuven Ramaty High Energy Solar Spectroscopic Imager (RHESSI) in 2002-2005. Using the CLEAN method, we derive the hard X-ray images and then extract light curves for each footpoint. We decompose the hard X-ray light curves into slowlyvarying components and fast-varying components using the Fourier method and check their correlations separately.

Results. Our analysis indicates that the asymmetry between hard X-ray footpoints is a ubiquitous feature in solar flares. However, in most asymmetric cases, the correlations between the light curves of the two footpoints remain high. In particular, the correlation coefficients between the slowly-varying components are significantly higher than those between the fast-varying components. We discuss the causes and implications of the asymmetries and correlations between hard X-ray footpoints.
\end{abstract}

Key words. Sun: flares - Sun: magnetic fields - Sun: X-rays, gamma rays

\section{Introduction}

The hard X-ray double-source structure is now thought to be a common feature in solar flares. Sakao (1994), for the first time, provided a statistical result on hard X-ray footpoint sources observed by the Yohkoh satellite and found that double sources are frequently observed. Physically, double hard X-ray sources are interpreted as caused by the precipitation of non-thermal electrons along the two legs of the magnetic loop (MacKinnon et al. 1985). According to the widely accepted CSHKP model (Carmichael 1964; Sturrock 1966; Hirayama 1974; Kopp \& Pneuman 1976), if the magnetic loop is symmetric and the reconnection site is just above the loop top, the non-thermal electron injection along the two legs should be the same, and one should observe two identical hard X-ray sources. However, observations always reveal two asymmetric sources (Sakao 1994; Kundu et al. 1995; Li et al. 1997; Aschwanden et al. 1999). Aschwanden et al. (1999) measured the footpoint distances and flux asymmetries for 54 Yohkoh/HXT flares with double sources. They established an asymmetric trap model to account for the observations. In this model, it was assumed that the acceleration site for the electrons is equidistant from each of the flare footpoints while the magnetic field strengths at the footpoints are different, resulting in different electron precipitation ratios. Siarkowski \& Falewicz (2004) studied in detail the asymmetry in an X5.3 flare of 25 August 2001 and found that it can even change with time.

The Reuven Ramaty High Energy Solar Spectroscopic Imager (RHESSI; Lin et al. 2002) has measured a large number of flares since its launch. The RHESSI data, with unprecedented spatial and temporal resolution, provide us with a good opportunity to investigate the asymmetries and correlations between two hard X-ray sources in detail. In this paper, we select a sample of 29 RHESSI flares with clearly defined double hard $\mathrm{X}$-rays sources and perform a statistical study. The results can be used to constrain the flare model, in particular, the properties in the energy release process.

The paper is organized as follows. In Sect. 2 we introduce the observational data with an emphasis on the selection of the flare sample. In Sect. 3 we describe the method for data analysis. Results and discussions are presented in Sect. 4, followed by conclusions in Sect. 5.

\section{Observations and selection of samples}

RHESSI has 9 detectors with different spatial resolutions. The software default collimators in making images are $4-8$. We add detector 3 to improve the spatial resolution. We do not use detector 2 because it over-resolves the maps and yields large noise. According to the detector parameters, the spatial resolution is $7 "$ with this choice. We check the images of solar flares above C-class during 2002-2005 based on GOES class. The images are made corresponding to the hard X-ray peak time in the $25-50 \mathrm{keV}$ energy band. This is because in the peak time, the double sources, if present, should be most clearly distinguishable. After this initial selection, we obtain 93 solar flares with double sources. Then we check the stability of the footpoints during the whole flare development. We remove the flares whose footpoints become dispersive or move fast with time ${ }^{1}$, where it is difficult to use fixed boxes to obtain hard X-ray fluxes at the footpoints. We also discard the ones with 3 or more footpoints during some periods of the evolution. After this process, only

\footnotetext{
1 The former refers to footpoint sources that have no well-defined edges and the latter to sources that move $\gtrsim 10^{\prime \prime}$ during the whole evolution (in several minutes).
} 
about one third of the flares remain. In order to confirm that the two sources correspond to the conjugate footpoints of the flare loop, we overlay the magnetogram observed by SOHO/MDI (Scherrer et al. 1995) on the RHESSI image to check the magnetic polarities. The conjugate footpoints are expected to lie on the two sides of the magnetic neutral line, so the polarities in them should be opposite. According to the polarity criterion, we obtain 29 flares (3 C-class, 18 M-class, 8 X-class) for study. In the sample, there are $3 \mathrm{limb}$ flares whose magnetic polarities do not conform strictly to this scenario. However, for these 3 events, we think that the projection effect is likely the main cause for the hard X-ray sources not meeting the polarity criterion. Therefore, we still keep them in the sample. We believe that the sample is an unbiased one that can represent all the flares with double footpoint sources. As an example, Fig. 1 shows 6 flares in this sample.

\section{Data analysis}

\subsection{Hard X-ray light curves at footpoints}

We choose two energy bands (25-50 and 50-100 keV) for this study. To derive the spatially resolved hard X-ray light curves, we need first to derive the hard X-ray images of the flaring region. The CLEAN method is adopted here (Hurford et al. $2002)^{2}$. In order to have a signal-to-noise ratio that is high enough, we select an integration time of $8 \mathrm{~s}$. We collect the images every $1 \mathrm{~s}$. This means that the integration time intervals for two consecutive images overlap by $7 \mathrm{~s}$. After having obtained the hard X-ray images, we can deduce the light curves for each footpoint. We draw fixed boxes encompassing the footpoints; the size and position of the boxes are chosen such that the footpoints (defined as $\geq 30 \%$ of the maximum hard X-ray emission) do not move out of them during the flare evolution. The pixel size of the light curves is $1 \mathrm{~s}$. However, the time resolution of such a curve is $8 \mathrm{~s}$, i.e., the integration time of the images. For each flare, the start time is defined as the beginning of the impulsive phase, while the end time is set to the moment when the flux falls to the background. In some flares, there appear several emission peaks. The light curves thus include all of them. In very few cases, the light curves may not be able to include the whole evolution of flares as caused by the Earth's shadow or the South Atlantic Anomaly (SAA), etc.

\subsection{Asymmetry of footpoints}

To quantify the asymmetries between the two conjugate footpoints in a flare, we define an asymmetry coefficient as

$A=\frac{F_{1}-F_{2}}{F_{1}+F_{2}}$

$F_{1}$ and $F_{2}$ represent the fluxes of the two footpoints respectively. Therefore, $A=0$ means the two footpoints are symmetric, while $A=1$ given the most extremely asymmetric case. The above formula is only applicable when the fluxes at the footpoints are above a critical value, which set to be $3 \sigma$ above the spatiallyintegrated pre-flare background. We can then calculate the

${ }^{2}$ The CLEAN algorithm reconstructs the RHESSI image in an iterative way that repeatedly subtracts from the dirty map (by Back Projection) a Point Spread Function until a negative value appears. In the initial stages of flares when the footpoints are still faint and cannot be clearly distinguished from the background, negative values of hard $\mathrm{X}$-ray flux are usually inevitable (see Fig. 3).
Table 1. The flare list.

\begin{tabular}{rcccccc}
\hline \hline Data & Class & Start & Peak & End & Location & NOAA \\
\hline 17-Mar.-2002 & M4.0 & $19: 24$ & $19: 31$ & $19: 34$ & S22E20 & 9871 \\
31-May-2002 & M2.4 & $00: 04$ & $00: 16$ & $00: 25$ & S18E59 & 9973 \\
3-Aug.-2002 & C5.3 & $12: 59$ & $13: 04$ & $13: 09$ & S09W14 & 0050 \\
10-Sep.-2002 & M2.9 & $14: 49$ & $14: 56$ & $15: 00$ & N15W14 & 0105 \\
20-Sep.-2002 & M1.8 & $09: 21$ & $09: 28$ & $09: 33$ & S03W27 & 0126 \\
29-Sep.-2002 & M2.6 & $06: 32$ & $06: 39$ & $06: 41$ & S06W55 & 0134 \\
9-Nov.-2002 & M4.6 & $13: 08$ & $13: 23$ & $13: 36$ & S12W29 & 0180 \\
10-Nov.-2002 & M2.4 & $03: 04$ & $03: 21$ & $03: 35$ & S12W37 & 0180 \\
29-May-2003 & X1.2 & $00: 51$ & $01: 05$ & $01: 12$ & S06W37 & 0365 \\
17-Jul.-2003 & C9.8 & $08: 17$ & $08: 23$ & $08: 29$ & N15E21 & 0412 \\
24-Oct.-2003 & M7.6 & $02: 27$ & $02: 54$ & $03: 14$ & S19E72 & 0486 \\
31-Oct.-2003 & C8.5 & $12: 24$ & $12: 31$ & $12: 35$ & N08W28 & 0488 \\
1-Nov.-2003 & M3.2 & $22: 26$ & $22: 38$ & $22: 49$ & S12W60 & 0486 \\
2-Nov.-2003 & X8.3 & $17: 03$ & $17: 25$ & $17: 39$ & S12W60 & 0486 \\
13-Nov.-2003 & M1.6 & $04: 54$ & $05: 01$ & $05: 06$ & S12W60 & 0486 \\
6-Jan.-2004 & M5.8 & $06: 13$ & $06: 29$ & $06: 36$ & N09W04 & 0528 \\
13-Jul.-2004 & M6.7 & $00: 09$ & $00: 17$ & $00: 23$ & S09W21 & 0646 \\
12-Sep.-2004 & M4.8 & $00: 04$ & $00: 56$ & $01: 33$ & N04E42 & 0672 \\
30-Oct.-2004 & M5.9 & $16: 18$ & $16: 33$ & $16: 37$ & N14W15 & 0691 \\
3-Nov.-2004 & M1.6 & $03: 23$ & $03: 35$ & $03: 57$ & N15W35 & 0691 \\
4-Nov.-2004 & M5.4 & $22: 53$ & $23: 09$ & $23: 26$ & N11E19 & 0696 \\
6-Nov.-2004 & M9.3 & $00: 11$ & $00: 34$ & $00: 42$ & N07E14 & 0696 \\
7-Nov.-2004 & X2.0 & $15: 42$ & $16: 06$ & $16: 15$ & N10W08 & 0696 \\
10-Nov.-2004 & X2.5 & $01: 59$ & $02: 13$ & $02: 20$ & N09W49 & 0696 \\
1-Jan.-2005 & X1.7 & $00: 01$ & $00: 31$ & $00: 39$ & N03E47 & 0715 \\
15-Jan.-2005 & X2.6 & $22: 25$ & $23: 02$ & $23: 31$ & N14W08 & 0720 \\
19-Jan.-2005 & X1.3 & $08: 03$ & $08: 22$ & $08: 40$ & S01E55 & 0720 \\
20-Jan.-2005 & X7.1 & $06: 36$ & $07: 01$ & $07: 26$ & S01E55 & 0723 \\
10-Sep.-2005 & X2.1 & $21: 30$ & $22: 11$ & $22: 43$ & S10E66 & 0808 \\
\hline & & & & & & \\
\hline
\end{tabular}

footpoint asymmetry for each flare. For our statistical study, we use time-integrated fluxes for $F_{1}$ and $F_{2}$.

\subsection{Correlation of footpoints}

The correlation coefficient between the two footpoints is then directly calculated based on their light curves. As above, we only use those parts of the light curves above $3 \sigma$ of the background. In order to distinguish between the fast and slow variations, we further decompose each light curve into a fast-varying component and a slowly-varying component. This can be done using the Fourier method. First, we use the Fast Fourier Transform to calculate the power spectrum of the light curves for each footpoint. Then, we split the power spectrum into a lower frequency domain and a higher frequency one. Lastly, we apply the Inverse Fast Fourier Transform to the two parts of the power spectrum to obtain slowly-varying and fast-varying components of the light curve respectively. The threshold timescale between the two components is arbitrarily chosen to be $32 \mathrm{~s}$. We cut off the high frequency variations with timescales less than $8 \mathrm{~s}$ in order to avoid the noise effect (see discussion in Sect. 3.4). Thus, the fast-varying component refers to variations with timescales in the range of 8-32 s, while the slowly-varying component refers to variations with timescales longer than $32 \mathrm{~s}$. Then, we can calculate the correlation coefficient between the fast-varying components and that between the slowly-varying components separately.

\subsection{Effect of noise}

As RHESSI is a satellite without a shield, the effect of the background and instrument noise in the data must be evaluated 

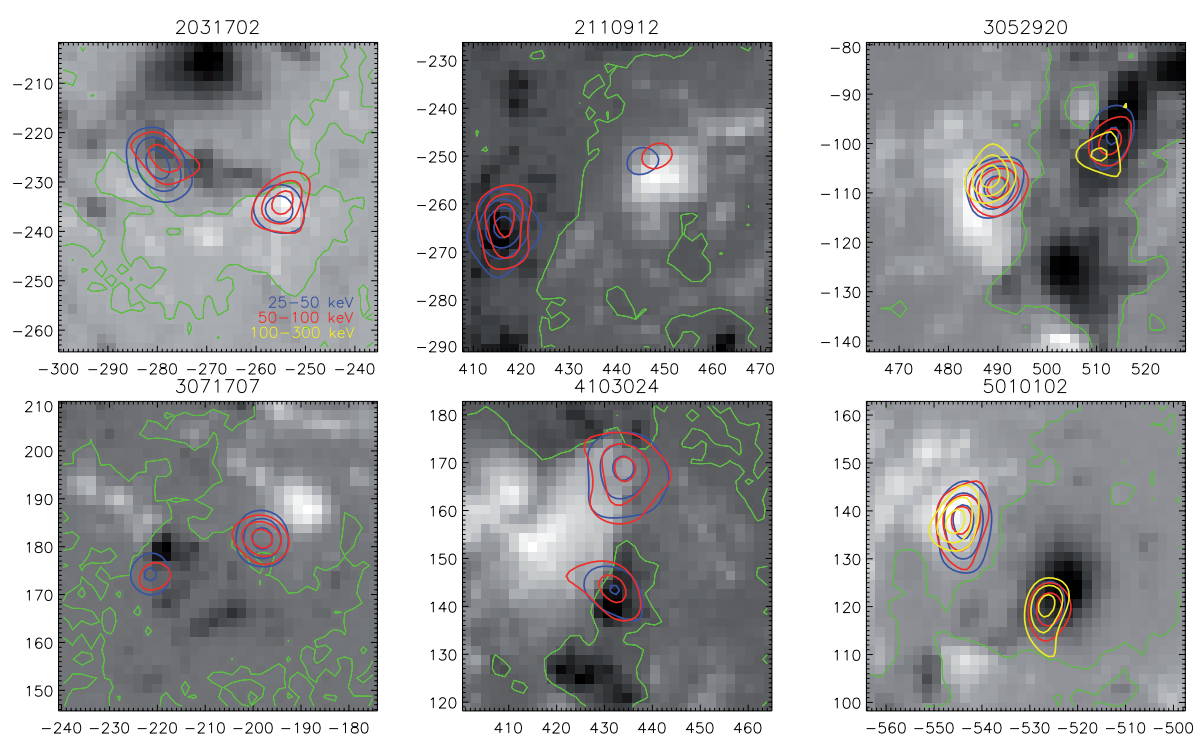

Fig. 1. Hard X-ray contours for 6 RHESSI flares, overlaid on the magnetograms observed by SOHO/MDI. The blue, red, and yellow contours represent $25-50,50-100,100-300 \mathrm{keV}$, respectively. The contour levels are $50 \%, 70 \%$, and $90 \%$. The green lines represent the magnetic neutral lines. to check the validity of our study (Smith et al. 2002). As shown above, the fluxes of the footpoints are deduced from the RHESSI CLEAN images. The first step is thus to estimate the uncertainties of the fluxes. Unfortunately, the error estimate of RHESSI images is still a open question, and there is not yet a standard way to determine this. Krucker \& Lin (2002) for the first time provided a way to estimate the error using residual maps made by the CLEAN algorithm. They take the maximum value of the flux outside the source as an approximation of the $3 \sigma$ error. Recently, Liu (2007) provided a similar way to estimate the error for a CLEAN image, using the rms of the residual map as the uncertainty of the value at each pixel. Liu then gave an error of about $10 \%$ of the source flux in low energies $(\sim 10 \mathrm{keV})$ and a slightly larger one in high energies. According to Liu's estimation, we assume a mean error of $15 \%$ in the $25-50 \mathrm{keV}$ and $20 \%$ in $50-100 \mathrm{keV}$ footpoint fluxes in our events. In the following, we use this error estimation to check its influence on the correlation coefficients of the footpoints.

Because the noise is caused by many factors, instrumental or from the numerical method, i.e., the image reconstruction method, we do not know if the noise at the two footpoints of a flare has a relationship or not. The worst case is that they are completely unrelated, which should have the largest influence on the correlation coefficients of the two footpoints derived by us. To test this, we assign two artificial and independent white noises, with their mean values as given above, to the two footpoint light curves of each flare. Then we repeat the method in Sect. 3.3 and calculate the correlation coefficients between the two footpoints. We find that the difference of the correlation coefficients in cases with and without artificial noises is less than $10 \%$. This means that the error does not significantly influence our statistical results.

We have filtered out the high-frequency variations with timescales of less than $8 \mathrm{~s}$ in our fast varying components. There are two reasons for this. One is that the noise effect on the correlation coefficients increases with increasing frequency. The other is the existence of pseudo-periodicities in the RHESSI hard $\mathrm{X}$-ray light curves due to the rotational modulation of the instrument. Generally we need to use the DEMODULATOR software to eliminate them. Unfortunately, this software can only be applied to the global light curve, but not the light curves for each footpoint. Considering that such pseudo-periodicities have timescales of $4 \mathrm{~s}$ or less, they should have been largely

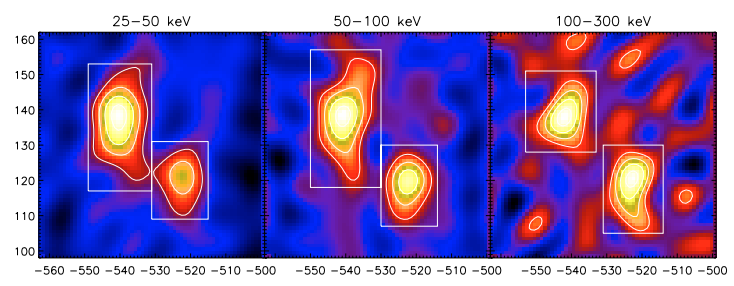

Fig. 2. RHESSI images of the 2005 January 5 flare at 00:26:20 UT. The two boxes, encompassing the two footpoints, represent the areas in which we derive the hard X-ray fluxes plotted in Fig. 3. The contour levels are $30 \%, 50 \%, 70 \%$, and $90 \%$.

smoothed out in the fast components derived here if we set a cutoff timescale of $8 \mathrm{~s}$.

\section{Results and discussion}

Using the above method, we study in detail the correlations between the footpoints. The results show that the slowly-varying components generally have a higher correlation coefficient than the fast-varying components. Figures 2 and 3 show an example for the 2005 January 5 flare. In Fig. 2, we plot the RHESSI images in the 25-50, 50-100, 100-300 keV derived with the CLEAN method. The two boxes, encompassing the two footpoints, represent the areas in which we derive the hard X-ray fluxes plotted in Fig. 3.

We can see from Fig. 3 that the fluxes at the two footpoints are highly correlated. After we decompose the light curves into slowly- and fast-varying components, we find that the slow ones still have a high correlation (correlation coefficient of 0.98), while the fast ones are much less correlated (correlation coefficient of 0.65 ).

Figure 4 plots the correlation coefficients of the slowlyvarying components versus those of the fast-varying components for the 29 flares in two energy bands (25-50 and 50-100 keV). In the $25-50 \mathrm{keV}$ band, the correlation coefficients of the slow components are usually high. For the fast components, the majority of them show low correlation coefficients, while some show relatively high correlation coefficients. The data points for $50-100 \mathrm{keV}$ are slightly more dispersed, possibly because of more noise in the higher energy band. In this band, there are very few cases showing a low correlation coefficient of 


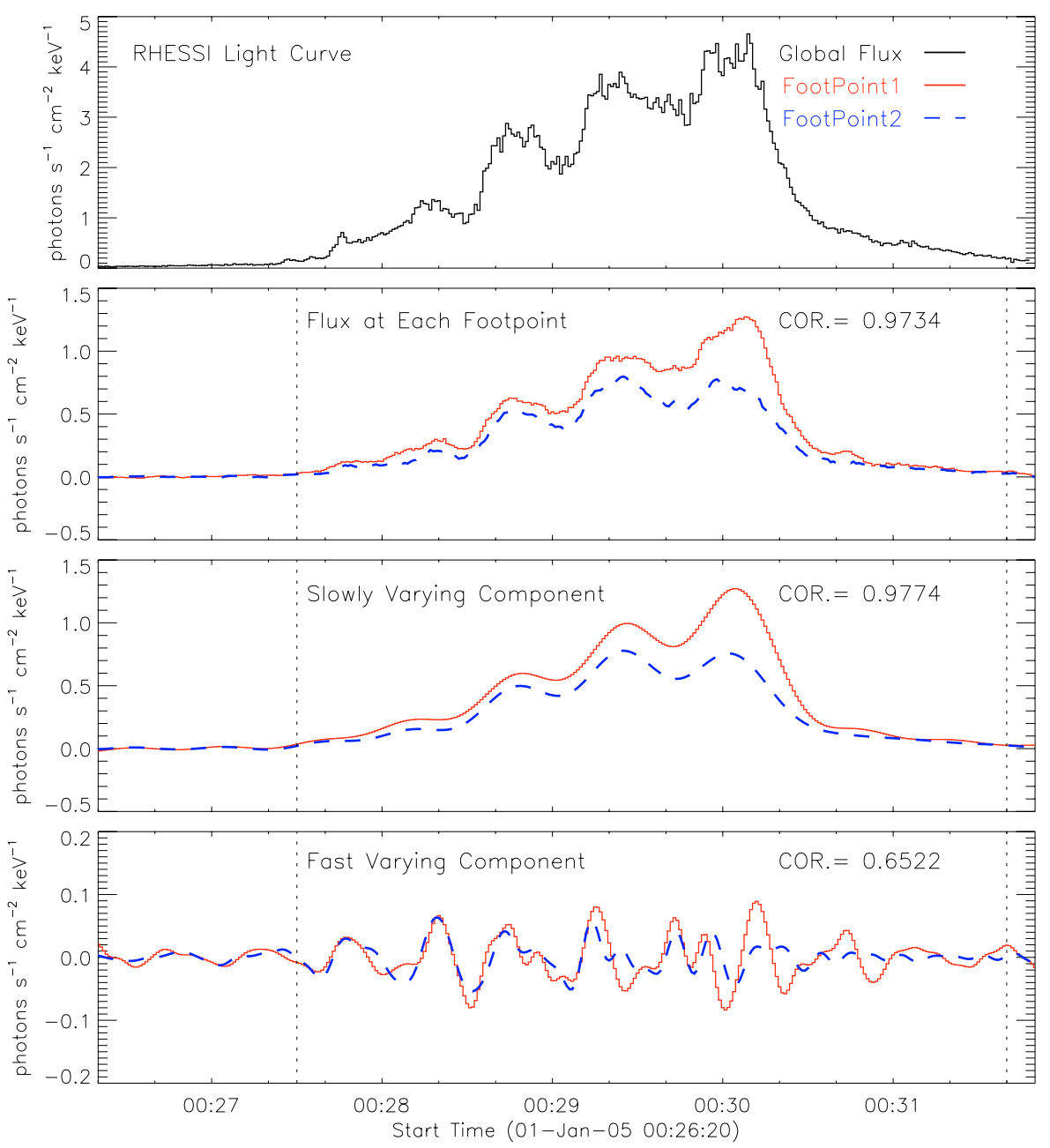

Fig. 3. Hard X-ray light curves at $50-100 \mathrm{keV}$ of the 2005 January 5 flare at 00:26:20 UT. Top panel: demodulated global flux of the Sun; second panel: fluxes at each footpoint; third panel: slowly-varying components; bottom panel: fast-varying components. The solid and dashed curves represent the two footpoints respectively. The dotted lines in the lower three panels represent the time interval for which we calculate the correlation coefficient.

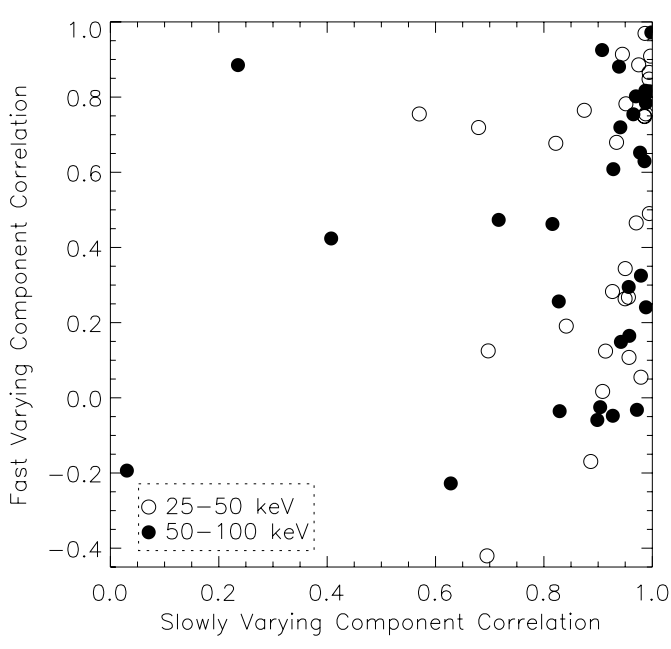

Fig. 4. Footpoint correlation coefficients of slowly varying components versus that of fast varying components.

the slow-varying components. There appears to be a slight energy-dependence of the results. However, the sample is not large enough to make this point conclusive. We need to check more events to establish if there really exists such an energydependence.

In Fig. 5, we plot the asymmetries of flare footpoints against their correlation coefficients. The footpoint asymmetries vary from case to case. However, the footpoint correlations are always

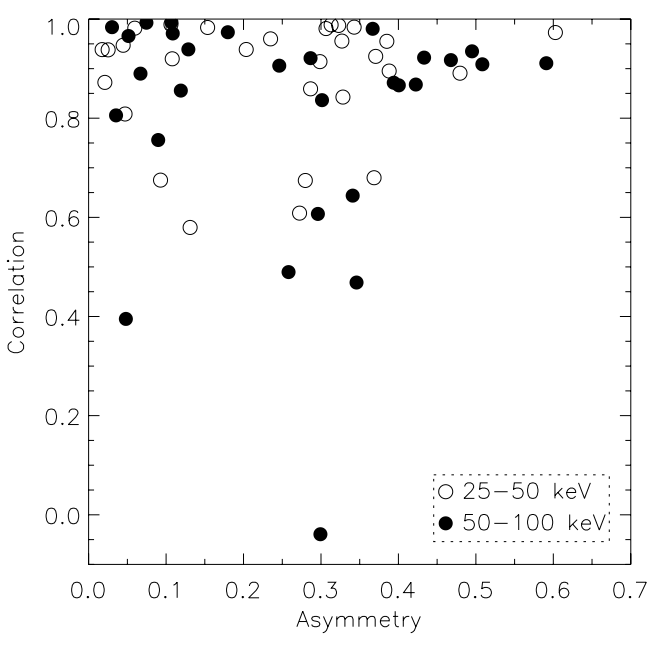

Fig. 5. Asymmetries versus correlation coefficients of flare footpoints.

high regardless of whatever asymmetries there are. Thus, even in the most asymmetric case, the footpoints still remain high correlated. There are very few exceptions, again in the $50-100 \mathrm{keV}$ band, in which the correlation is weak.

There are several interpretations about the asymmetries between the two conjugate footpoints. One possible cause is the magnetic mirroring effect (e.g., Sakao 1994; Aschwanden et al. 1999). In the footpoint with a stronger magnetic field, more 
electrons are reflected by magnetic mirroring back into the top of the loop, while fewer electrons can directly precipitate into deeper layers. Therefore, a brighter hard X-ray source is usually expected to correspond to a weak magnetic field and vice versa. However, some recent observations showed that such a scenario is not always true (Asai et al. 2002; Goff et al. 2004). The brighter hard X-ray footpoints sometimes coincide with the regions of stronger magnetic fields. Goff et al. (2004) designated these flares as N-type ones and found that the occurrence ratio of such flares is as high as one third.

Another possibility leading to footpoint asymmetries is asymmetric magnetic reconnection. If the reconnection site is not located at the mid-point between the two footpoints but closer to one of them, then the electrons released at the reconnection site should pass through different column densities before they reach the chromosphere in the two footpoints. This results in different hard X-ray sources. Emslie et al. (2003) found evidence of the different column densities implied by a difference in spectral indices of the footpoints. Such a model also has been investigated in detail by Falewicz \& Siarkowski (2007). Through numerical calculations, they found that an asymmetric electron injection site, together with different mass densities in the loop, can quantitatively account for the observed hard X-ray asymmetries.

The high correlations between the conjugate footpoints, as revealed by the hard X-ray light curves, suggest that they are physically linked irrespective of the (possibly) quite different circumstances in the two footpoints that cause different hard $\mathrm{X}$-ray fluxes. The reason why we decompose the light curves into slowly- and fast-varying components is that we need to check what timescale such correlations exist. We have found that the correlations are higher in slow components than in fast components by setting a cutoff timescale of $32 \mathrm{~s}$. However, this timescale is arbitrary. We have changed it in the range of 10-80 s and found that such a rule is always valid.

Fine time structures in HXR emission have been reported (e.g., Kiplinger et al. 1983, 1984; Dennis 1986; Aschwanden et al. 1995, 1998). Similar fine structures have also been found in microwave emissions (e.g., Slottje 1978; Takakura et al. 1983; Elgaroy 1986; Kliem et al. 2000; Nakajima 2000) and even in optical emissions (Wang et al. 2000; Ding et al. 2001; Ding 2005). They are all thought to be related to the time characteristics of electron beams. However, the physical origin is still unclear. Aschwanden (2004) pointed out that the time characteristics are controlled by the injection mechanism, i.e., the impulsive bursty reconnection regime, rather than by the overall acceleration mechanism. An assumption is that the fine structures in the hard X-ray time profile correspond to small-scale electron injections, and that the slow components are build up by a large number of such small bursts. Then, the relatively low correlations between the fast hard X-ray components in the two footpoints imply that the injections are not evenly distributed along the two legs. However, the high correlations between the slow components suggest that the time integrations of such injections, on long timescales, are still correlated.

Such a scenario is consistent with a turbulent model proposed by Jakimiec (1998). In this model, electrons can be independently injected into each leg, resulting in different fine structures in the hard X-ray light curves of the two footpoints.

\section{Conclusions}

We chose 29 solar flares observed by RHESSI during 2002-2005 with double hard X-ray footpoints for a statistical study of the footpoint asymmetries and correlations. Using the CLEAN method, we reconstructed the hard X-ray images and then extract the light curves for each footpoint. We used the Fourier method to divide the light curve into a slowly-varying component and a fast-varying component. Our main conclusions are as follows:

1. There is usually a good correlation between the light curves of the two conjugate footpoints in a flare. However, the correlations for the slow components are much better than those for fast components.

2. Asymmetries between hard X-ray footpoints are a common phenomenon in solar flares. However, in most asymmetric cases, the correlations between the footpoints remain high.

3. The results suggest a scenario that the small injections of electrons are independent in the two footpoints, while the integration of them (revealed by the slow component in the light curve) still keeps a high correlation.

Acknowledgements. We thank Q. R. Chen for providing the flare list and valuable discussions and G. Hurford for help in RHESSI data analysis. We are very grateful to the referee for valuable comments that helped improve the paper. This work was supported by National Natural Science Foundation of China (NSFC) under grants $10025315,10221001,10333040$, and 10673004, and by NKBRSF under grant 2006CB806302.

\section{References}

Asai, A., Masuda, S., Yokoyama, T., et al. 2002, ApJ, 578, L91

Aschwanden, M. J. 2004, ApJ, 608, 554

Aschwanden, M. J., Schwartz, R. A., \& Alt, D. M. 1995, ApJ, 447, 923

Aschwanden, M. J., Kliem, B., Schwarz, U., et al. 1998, ApJ, 505, 941

Aschwanden, M. J., Fletcher, L., Sakao, T., Kosugi, T., \& Hudson, H. 1999, ApJ, 517,977

Carmichael, H. 1964, in The Physics of Solar Flares, ed. W. N. Hess, 451

Dennis, B. R. 1986, in Solar Flares and Coronal Physics Using P/OF as a Research Tool, ed. R. M. Wilson, \& H. S. Hudson (NASA CP-2421; Washington, DC: NASA), 67

Ding, M. D. 2005, Adv. Space Res., 35, 1846

Ding, M. D., Qiu, J., Wang, H., \& Goode, P. R. 2001, ApJ, 552, 340

Elgaroy, O. 1986, Sol. Phys., 104, 43

Emslie, A. G., Kontar, E. P., Krucker, S., \& Lin, R. P. 2003, ApJ, 595, L107

Falewicz, R., \& Siarkowski, M. 2007, A\&A, 461, 285

Goff, C. P., Matthews, S. A., van Driel-Gesztelyi, L., \& Harra, L. K. 2004, A\&A, 423,363

Hirayama, T. 1974, Sol. Phys., 34, 323

Hurford, G. J., Schmahl, E. J., Schwartz, R. A., et al. 2002, Sol. Phys., 210, 61

Jakimiec, J. 1998, Publ. Astron. Inst. Czechoslovak Academy Sci., 88, 124

Kiplinger, A. L., Dennis, B. R., Frost, K. J., Orwig, L. E., \& Emslie, A. G. 1983, ApJ, 265, L99

Kiplinger, A. L., Dennis, B. R., Frost, K. J., \& Orwig, L. E. 1984, ApJ, 287, L105

Kliem, B., Karlický, M., \& Benz, A. O. 2000, A\&A, 360, 715

Kopp, R. A., \& Pneuman, G. W. 1976, Sol. Phys., 50, 85

Krucker, S., \& Lin, R. P. 2002, Sol. Phys., 210, 229

Kundu, M. R., Nitta, N., White, S. M., et al. 1995, ApJ, 454, 522

Li, J., Metcalf, T. R., Canfield, R. C., Wuelser, J.-P., \& Kosugi, T. 1997, ApJ, 482,490

Lin, R. P., Dennis, B. R., Hurford, G. J., et al. 2002, Sol. Phys., 210, 3

Liu, W. 2007, Ph.D. Thesis, Stanford University

MacKinnon, A. L., Brown, J. C., \& Hayward, J. 1985, Sol. Phys., 99, 231

Nakajima, H. 2000, in High Energy Solar Physics Workshop: Anticipating HESSI, ed. R. Ramaty, \& N. Mandzhavidze (San Francisco: ASP), ASP Conf. Ser., 206, 313

Sakao, T. 1994, Ph.D. Thesis, University of Tokyo

Scherrer, P. H., Bogart, R. S., Bush, R. I., et al. 1995, Sol. Phys., 162, 129

Siarkowski, M., \& Falewicz, R. 2004, A\&A, 428, 219

Slottje, C. 1978, Nature, 275, 520

Smith, D. M., Lin, R. P., Turin, P., et al. 2002, Sol. Phys., 210, 33

Sturrock, P. A. 1966, Nature, 211, 695

Takakura, T., Nitta, N., Kaufmann, P., et al. 1983, Nature, 302, 317

Wang, H., Qiu, J., Denker, C., et al. 2000, ApJ, 542, 1080 